\title{
Concurrent study of bottomside spread $F$ and plasma bubble events in the equatorial ionosphere during solar maximum using digisonde and ROCSAT-1
}

\author{
C.-C. Lee $^{1}$, S.-Y. Su ${ }^{2}$, and B. W. Reinisch ${ }^{3}$ \\ ${ }^{1}$ General Education Center, Ching-Yun University, Jhongli City, Taoyuan County, Taiwan \\ ${ }^{2}$ Institute of Space Science and Center for Space and Remote Sensing Research, National Central University, Jhongli City, \\ Taoyuan County, Taiwan \\ ${ }^{3}$ Center for Atmospheric Research, University of Massachusetts Lowell, Massachusetts, USA
}

Received: 18 July 2005 - Revised: 7 October 2005 - Accepted: 26 October 2005 - Published: 21 December 2005

\begin{abstract}
Data from the Jicamarca digisonde and the ROCSAT-1 satellite are employed to study the equatorial ionosphere on the west side of South America during April 1999-March 2000 for the concurrent bottomside spread F (BSSF) and plasma bubble events. This study, using digisonde and ROCSAT- 1 concurrently, is the first attempt to investigate the equatorial spread F. Results show that BSSF and plasma bubble observations appear frequently respectively in the summer (January, February, November, and December) and in the equinoctial (March, April, September, and October) months, respectively, but are both rarely observed in the winter (May-August) months. The upward drift velocity during the concurrent BSSF and bubble observations has been determined to study the driving mechanism. This analysis shows that large vertical drift velocities favor BSSF and bubble formations in the equinoctial and summer months. Conversely, the smaller upward velocities during the winter months cause fewer BSSF and bubble occurrences. For the geomagnetic effect, the BSSF/bubble occurrence decreases with an increasing $K_{p}$ value in the equinoctial months, but no such correlation is found for the summer and winter months. Moreover, the anti-correlations between $K_{p}$ and $\mathrm{d} h^{\prime} F / \mathrm{dt}$ are apparent in the equinoctial months, but not in the summer and winter months. These results indicate that in the equinoctial months the BSSF/bubble generations and the pre-reversal drift velocity can be suppressed by geomagnetic activity, because the disturbance dynamo effects could have decreased the eastward electric field near sunset. However, BSSF and bubble occurrences may not be suppressed by the geomagnetic activity in the summer and winter months.
\end{abstract}

Keywords. Ionosphere (Ionospheric irregularities; Equatorial ionosphere)

Correspondence to: C.-C. Lee

(cclee@cyu.edu.tw)

\section{Introduction}

Plasma irregularities and inhomogeneities in the equatorial $\mathrm{F}$ region caused by plasma instabilities are called the equatorial spread F (ESF) (e.g. Kelley, 1989). Many investigators (Tsunoda et al., 1982; Zalesack et al., 1982; Kelley, 1989; Sultan, 1996) have proposed that the instability processes, involving the gravitational Rayleigh-Taylor (GRT) and $\boldsymbol{E} \times \boldsymbol{B}$ instabilities, could have started with irregularities in the bottomside F-layer (bottomside spread F, BSSF). Further, the nonlinear development of instabilities will form the vertical elongated wedges of plasma depletions (bubbles) that drift upward from the bottomside F-layer to altitudes as high as 1500 km (e.g. Kelley, 1989; Sultan, 1996). According to the previous investigations (e.g. Aarons, 1993; Whalen, 2002), the irregularities of the equatorial BSSF are usually confined to below the F-peak. Therefore, BSSF, which maps via the geomagnetic field to a narrow band of latitude, is observed only by sounders near the dip equator. On the other hand, plasma bubbles can extend to a wider latitudinal coverage, because the flux tubes in a vertical wedge are typical depleted along their north-south extent (Whalen, 1997, 2002).

Since the original description of the equatorial BSSF and bubble by Woodman and La Hoz (1976), the two have been interesting subjects of many studies (e.g. Whalen, 1997, 2002; Fejer et al., 1999; Huang, 2001; Su et al., 2001). The past results indicate that the occurrences of both BSSF and bubble depend on season, longitude, solar cycle, and geomagnetic activity (Maruyama and Matuura, 1984; Whalen, 1997, 2002; Fejer et al., 1999; Huang, 2001; Lee at al., 2005). Nevertheless, little work has been published examining the simultaneous occurrence of BSSF and bubble in the equatorial ionosphere (Whalen, 1997, 2002). In his first report, Whalen (1997) compared the monthly occurrence of bubble with that of BSSF during solar maximum, using a chain of ionospheric sounders in the western American sector. He found that the BSSF and bubble occurrences were 


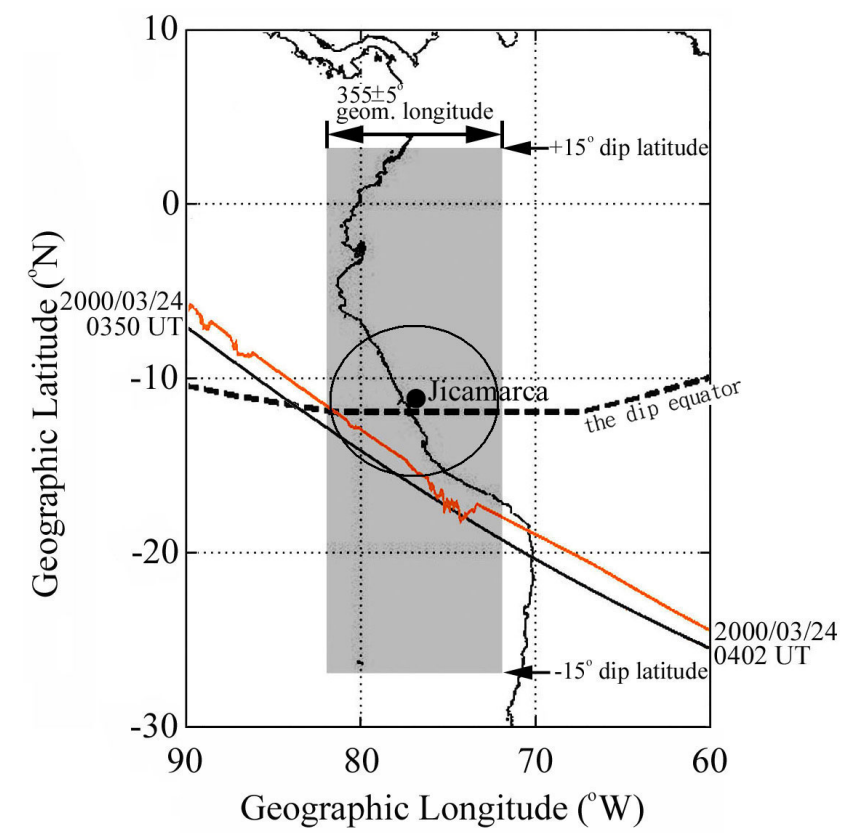

Fig. 1. The location of Jicamarca digisonde (black dot) and the area for ROCSAT-1 data selection (shaded area). The solid circle represents the wide beam of the digisonde transmit antenna at 600$\mathrm{km}$ altitude. The shaded area is through $\pm 15^{\circ}$ dip latitudes and $355 \pm 5^{\circ}$ geomagnetic longitudes. An example of a ROCSAT- 1 pass (solid line) and the associated ion density measurement (red line) is during 03:50-04:02 UT on 24 March 2000.

greater in the summer (December) and equinoctial (April and September) months, and had a minimum in the winter (July) months. Then Whalen (2002) employed the same data set to study the dependence of BSSF and bubble occurrences on season, the geomagnetic activity, and the $\boldsymbol{E} \times \boldsymbol{B}$ drift velocity. He suggested that bubble occurrence is associated with the magnitude of the drift velocity, and the BSSF/bubble generation is suppressed by the geomagnetic activity.

Although Whalen $(1997,2002)$ simultaneously investigated the BSSF and bubble formations in the equatorial ionosphere, he did not use satellite data or estimated the vertical drift velocity from his data set. The vertical drift velocity has been known to greatly affect the occurrences of the BSSF and plasma bubble (Fejer et al., 1999). Therefore, to study the BSSF and bubble more thoroughly, we employ the Jicamarca $\left(12^{\circ} \mathrm{S}, 76.9^{\circ} \mathrm{W}\right.$, dip latitude: $\left.1.2^{\circ} \mathrm{N}\right)$ digisonde data and the ROCSAT- 1 observations of plasma bubbles, to study the equatorial ionosphere from April 1999 to March 2000. In particular, the vertical $\boldsymbol{E} \times \boldsymbol{B}$ drift derived from the minimum virtual height of the F-layer $\left(h^{\prime} F\right)$ on the observed ionogram is used in this study. Moreover, we have used these data to examine the dependence of the BSSF and bubble occurrences on season, the $\boldsymbol{E} \times \boldsymbol{B}$ drift velocity, and the geomagnetic activity $\left(K_{p}\right)$ in the current study.
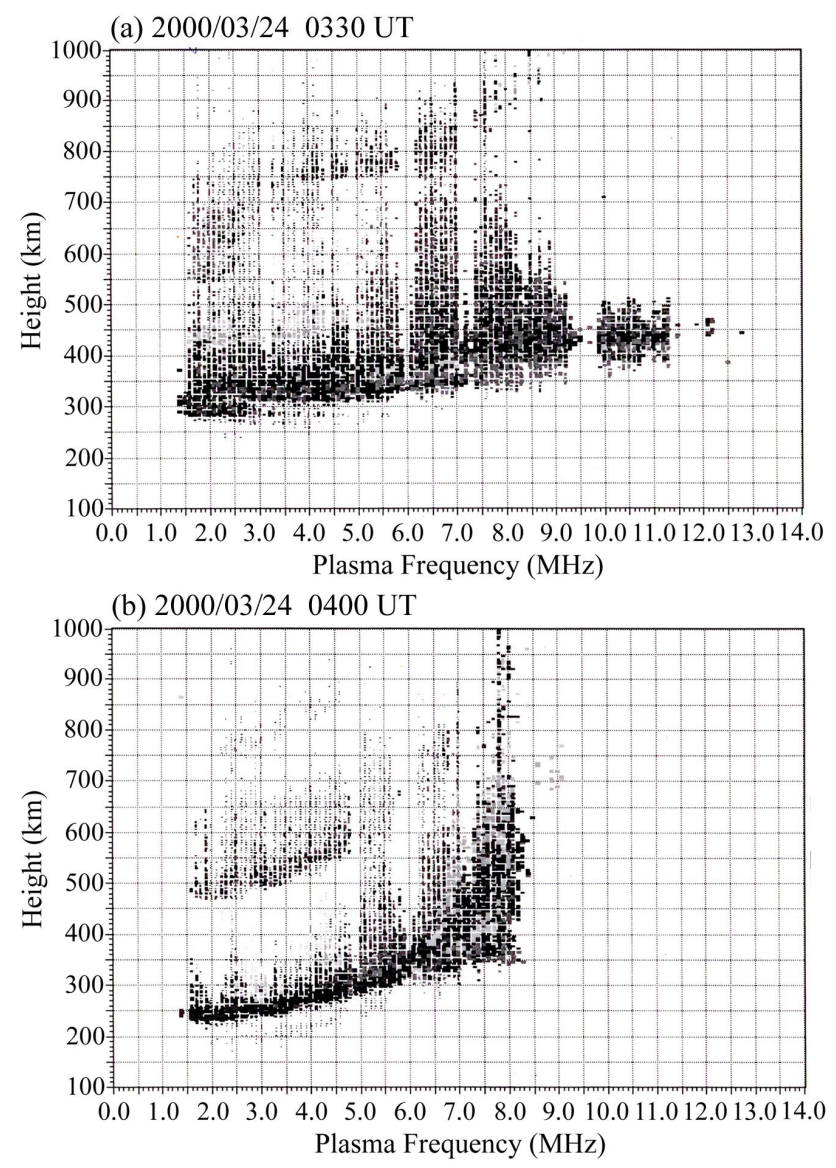

Fig. 2. Two ionogram examples of Jicamarca BSSF at (a) 03:30 and (b) 04:00 UT on 24 March 2000.

\section{Experiment setup}

The Jicamarca digisonde $\left(12^{\circ} \mathrm{S}, 76.9^{\circ} \mathrm{W}\right.$, dip latitude: $1.2^{\circ} \mathrm{N}$, geomagnetic longitude: $355^{\circ}$, see Fig. 1) observes the ionosphere near the dip equator. The solid circle in Fig. 1 represents a wide beam illuminating an area of $\sim 500-\mathrm{km}$ radius at $600 \mathrm{~km}$ altitude of the digisonde transmit antenna. The ionogram data with a time interval of $30 \mathrm{~min}$ during April 1999-March 2000, which is close to the peak of solar cycle 23 which is in April 2000, are used for this study. Notice that in this period, the monthly smoothed sunspot numbers are between $85.5-119.9$ and the monthly average values of F10.7 solar flux are 118.0-206.1. The presence/absence of a BSSF and other ionospheric parameters, for example, $h^{\prime} F$ (minimum virtual height of the $\mathrm{F}$ layer), foF2 (maximum frequency of F-layer), RF (range spread in $\mathrm{km}$ ), were obtained by the ARTIST program (Reinisch, 1996) and manual works. For example, two ionograms of BSSF at 03:30 and 04:00 UT of 24 March 2000 are displayed in Figs. 2a and $b$, respectively. Because we focus on the pre-midnight BSSF, only BSSF that occurred between 18:00 and 24:00 LT (LT=UT-5 h) is included in the statistical analysis. Moreover, we deduced the value of $\mathrm{d} h^{\prime} F / \mathrm{dt}$ from the temporal rate (the time interval is $30 \mathrm{~min}$ ) of $h^{\prime} F$. Notably, the value 


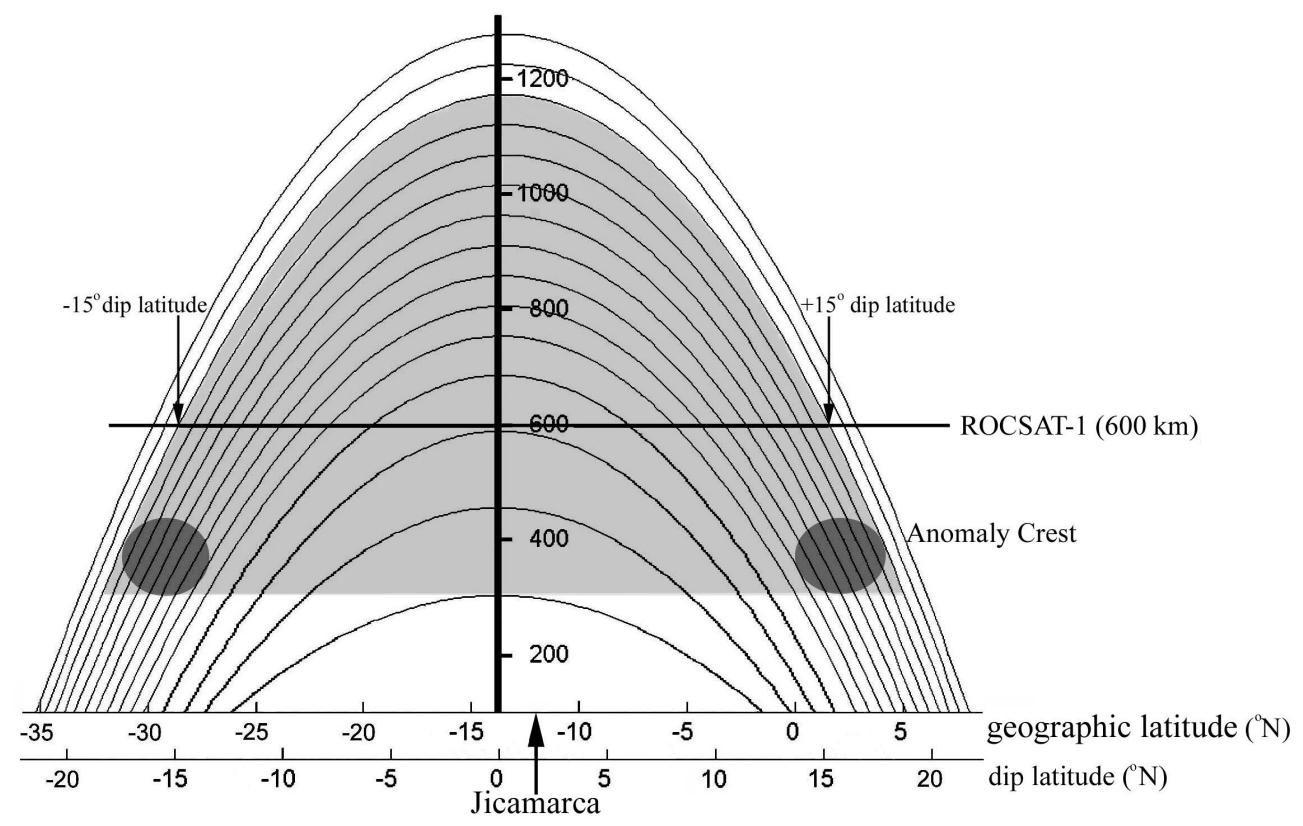

Fig. 3. Illustration of the bubble distribution in altitude and in relation to the geomagnetic fields. An equatorial bubble can rise to high altitudes and extent via the geomagnetic field to high latitudes as $\sim 16^{\circ}$ dip latitude (gray area) where the crest of equatorial ionization anomaly is located. When a bubble intersects the anomaly crest, it is detectable at $600 \mathrm{~km}$ between $\pm 15^{\circ}$ dip latitude by the ROCSAT- 1 .

Table 1. Numbers of BSSF day, digisonde day, bubble day, and ROCSAT-1 day for each month during April 1999-March 2000.

\begin{tabular}{lllllllllllll}
\hline month & Jan & Feb & Mar & Apr & Mai & Jun & Jul & Aug & Sep & Oct & Nov & Dec \\
\hline BSSF day (days) & 17 & 26 & 30 & 20 & 12 & 7 & 9 & 14 & 21 & 21 & 25 & 23 \\
digisonde day (days) & 17 & 28 & 31 & 26 & 27 & 22 & 20 & 29 & 26 & 25 & 26 & 24 \\
\hline bubble day (days) & 6 & 9 & 9 & 3 & 0 & 0 & 0 & 1 & 4 & 6 & 3 & 5 \\
ROCSAT-1 day (days) & 16 & 25 & 17 & 15 & 12 & 12 & 12 & 14 & 12 & 13 & 11 & 20 \\
\hline
\end{tabular}

of $\mathrm{d} h^{\prime} F / \mathrm{dt}$ can describe the $\boldsymbol{E} \times \boldsymbol{B}$ drift velocity, according to Bittencourt and Abdu (1981). Since $\boldsymbol{E} \times \boldsymbol{B}$ drift generally reaches its maximum value before the onset of a BSSF (e.g. Fejer et al., 1999; Whalen, 2002; Lee et al., 2005), we utilize the maximum value of $\mathrm{d} h^{\prime} F / \mathrm{dt}$ (hereafter described as $\mathrm{d} h^{\prime} F / \mathrm{dt}$ ) between 18:00 LT and the time of BSSF onset in the following analysis.

Bubbles are recorded by the ion trap (IT) on board ROCSAT- 1 orbiting at a $600-\mathrm{km}$ altitude with a $35^{\circ}$ inclination (see Yeh et al. (1999) for detail). For a concurrent study with the Jicamarca BSSF, we chose the plasma bubbles that appear in the area inside $\pm 15^{\circ}$ dip latitudes and $355 \pm 5^{\circ}$ geomagnetic longitudes (shaded area in Fig. 1) during 18:0024:00 LT. The $\pm 15^{\circ}$ dip latitude limit is chosen because it is near the location of the crests of the equatorial ionization anomaly in both the north and south hemispheres (dark ellipse in Fig. 3) where bubbles can be extended via the geomagnetic field (Whalen, 1997, 2002). The region of $355 \pm 5^{\circ}$ geomagnetic longitudes is chosen because of the coverage of the beam width of the Jicamarca digisonde. Therefore, a bubble that appears in the gray area of Fig. 1 during premidnight can be associated with bubbles that are over the top of the Jicamarca BSSF. An example of a ROCSAT-1 bubble (red line) that appears simultaneously with the Jicamarca BSSF (shown in Fig. 2) and the associated satellite pass (solid line) during 03:50-04:02 UT on 24 March 2000 are shown in Fig. 1.

\section{Results and discussion}

\subsection{Seasonal occurrences of BSSF and bubble formation}

In each month, the number of days (BSSF day) on which at least one pre-midnight BSSF event is observed on that day and the number of days of digisonde observation (digisonde day) are shown in Table 1. For the bubble events, the number of days (bubble day) on which at least one pre-midnight bubble event is observed in the chosen area (Fig. 1) on that day and the number of days (ROCSAT-1 day) on which the 


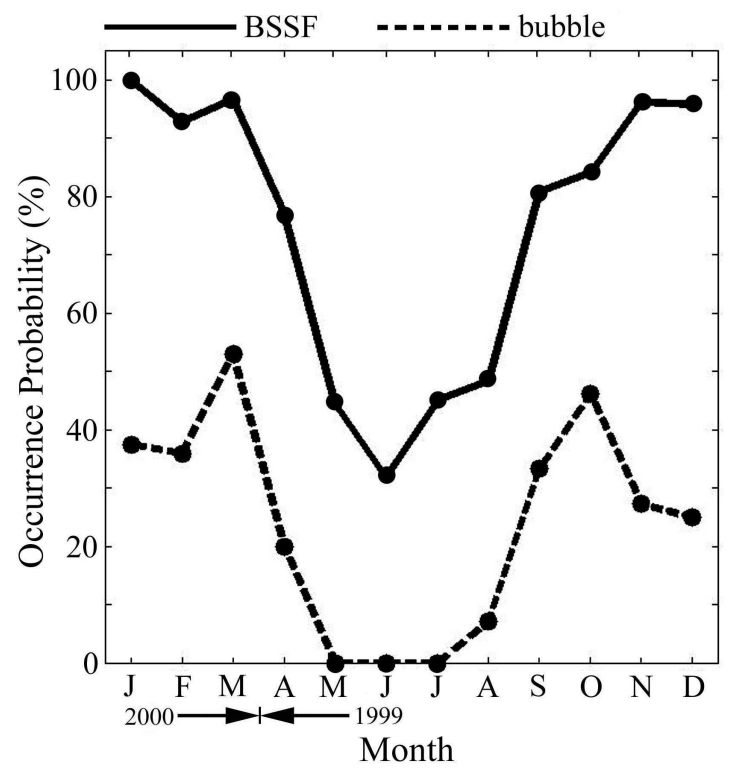

Fig. 4. The seasonal variations in the BSSF (solid line) and bubble (dashed line) occurrences during March 1999-April 2000.

ROCSAT- 1 passes over the chosen area during pre-midnight are also displayed in Table 1.

Figure 4 shows the seasonal variation in the occurrence probabilities of BSSF (solid line) and bubble (dashed line) between April 1999 and March 2000. The occurrence probability of BSSF is the ratio of BSSF day to digisonde day. For the occurrence of BSSF, the highest (100\%) and lowest (32\%) probabilities of occurrence fall in January (summer) and in June (winter), respectively. During the equinoctial months (March, April, September, and October), the occurrence probabilities are more than $75 \%$. Generally, the BSSF occurrence is higher in the summer (November, December, January, and February)/the equinoctial months, than in the winter months (May, June, July, and August). The seasonal variation of the Jicamarca BSSF occurrence is similar with that in the Huancayo $\left(12^{\circ} \mathrm{S}, 75.3^{\circ} \mathrm{W}\right) \mathrm{BSSF}$ occurrence rate reported by Whalen (2002) for 1958. He found that the highest and lowest occurrence rates of total BSSF (his Fig. 4) are in December and July, respectively.

For the bubble event, the occurrence probability is the ratio of bubble day to ROCSAT-1 day. It is found that in Fig. 4 the highest occurrence probability for bubbles does not exist in the summer months. Two peaks of high bubble occurrence probability are noticed in March (53\%) and October (46\%), with the lowest occurrence in the winter months. Notably, only one bubble has been observed by ROCSAT- 1 in August and none during May-July (0\%). Such distribution for the two-peak variation in bubble occurrence has also been proposed by Huang et al. (2001) and Whalen (2002). Huang et al. (2001) showed that DMSP F9 measured the highest occurrences of the equatorial plasma bubble over Huancayo (their Fig. 7) in March and October. In addition, Whalen (2002) concluded that the two peaks of bubble occurrence

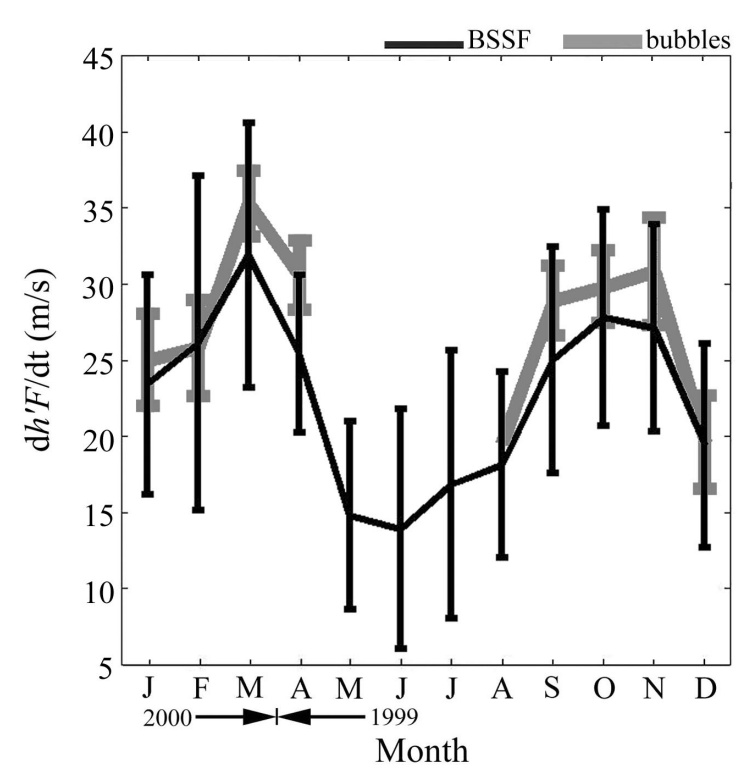

Fig. 5. The seasonal variations in the monthly mean $\mathrm{d} h^{\prime} F / \mathrm{dt}$ of the BSSF (black line) and bubble (gray line) events. The error bar represents the standard deviation of the mean $\mathrm{d} h^{\prime} F / \mathrm{dt}$ in the BSSF and bubble events.

are in April and September (his Fig. 4). It is now known that the seasonal variation in the BSSF occurrence is related to the magnitude of the maximum pre-reversal drift velocity and the late reversal time of the vertical velocity (Fejer et al., 1999; Whalen, 2002). However, the reason for the seasonal variation in the bubble occurrence is still not clear. Although Whalen (2002) suggested that the bubble occurrence is associated with the magnitude of the maximum pre-reversal drift velocity, he did not compare the probability of bubble occurrence with the vertical drift velocity of his data set. Thus, it is necessary to study the relationship between the BSSF/bubble occurrence and the associated vertical drift velocity.

\subsection{Seasonal variation in $\mathrm{d} h^{\prime} F / \mathrm{dt}$}

In this section, we examine the seasonal variations in the monthly mean value of $\mathrm{d} h^{\prime} F / \mathrm{dt}$ (Fig. 5). The mean value of $\mathrm{d} h^{\prime} F / \mathrm{dt}$ for the BSSF (black line)/bubble (gray line) events in a month is the average of the maximum pre-reversal drift velocities of days on which at least a pre-midnight $\mathrm{BSSF} /$ bubble is observed. For the BSSF event, the mean $\mathrm{d} h^{\prime} F / \mathrm{dt}$ peaks in March $(32 \mathrm{~m} / \mathrm{s})$ and in October $(28 \mathrm{~m} / \mathrm{s})$. During the summer and winter months, the mean $\mathrm{d} h^{\prime} F / \mathrm{dt}$ are $20-27$ and $14-18 \mathrm{~m} / \mathrm{s}$, respectively. Regarding the bubble event, there are also two peaked values found, one in March $(35 \mathrm{~m} / \mathrm{s})$ and the other in October $(33 \mathrm{~m} / \mathrm{s})$. The values of the mean $\mathrm{d} h^{\prime} F / \mathrm{dt}$ are $20-31$ and $20 \mathrm{~m} / \mathrm{s}$ in the summer months and in August, respectively. During the months from May to July, no $\mathrm{d} h^{\prime} F / \mathrm{dt}$ is calculated because no bubble appeared in these months. These seasonal variations are close to what Fejer et al. (1999) found (his Fig. 8). They claimed that the largest and smallest vertical velocities are 
found at the equinox and the June solstice, respectively. The values of the maximum vertical velocity during the high solar activity period obtained by Fejer et al. (1999) are 33-48, $28-34$, and $18 \mathrm{~m} / \mathrm{s}$ in the equinoctial, summer, and winter months, respectively. Notably, in Fig. 5, the values of mean $\mathrm{d} h^{\prime} F / \mathrm{dt}$ (standard deviation) are generally larger (smaller) for the bubble event than for the BSSF event.

For the BSSF events, the seasonal variation in the mean $\mathrm{d} h^{\prime} F / \mathrm{dt}$ is different from that in the BSSF occurrence (solid line in Fig. 4). During the equinoctial months, a larger $\mathrm{d} h^{\prime} F / \mathrm{dt}$ and a higher BSSF occurrence rate indicate that a larger upward drift lifts the F-layer to higher altitudes, which not only results in a favorable condition for the GRT instability but also causes an $\boldsymbol{E} \times \boldsymbol{B}$ drift instability (Maruyama, 1988; Kelley, 1989). In contrast, the small $\mathrm{d} h^{\prime} F / \mathrm{dt}$ value and the lower occurrence in the winter months suggest that the F-layer is not raised to an altitude high enough to generate instabilities. These relations between the mean value of $\mathrm{d} h^{\prime} F / \mathrm{dt}$ and the BSSF occurrence distribution indicate that the pre-reversal $\boldsymbol{E} \times \boldsymbol{B}$ drift plays an important role in the seeding mechanism of developing BSSF (Sultan, 1996; Fejer et al., 1999; Whalen, 2002; Lee et al., 2005). Additionally, during the summer months, the highest BSSF occurrence and the mean values of $\mathrm{d} h^{\prime} F / \mathrm{dt}, 20-27 \mathrm{~m} / \mathrm{s}$, suggest that another mechanism would also help the BSSF formation. In addition, the late reversal time of the upward drift velocity should be another reason to account for the highest occurrence probability at December solstice, because the late reversal time is favorable for the development of strong scattering layers in the F-region (Maruyama, 1988; Fejer et al., 1999).

For the bubble events, the mean $\mathrm{d} h^{\prime} F / \mathrm{dt}$ and the probability occurrence (dashed line in Fig. 4) both show peaks occurring in March and October. This coincidence between the two demonstrates that the magnitude of the maximum pre-reversal velocity is a principle mechanism for the bubble generation. Furthermore, the result obtained in this observation supports the report of Whalen (2002), who compared the bubble occurrence with the maximum pre-reversal drift velocity of Fejer et al. (1999).

3.3 Geomagnetic effect on the occurrence of BSSF and bubble formation

The occurrence of BSSF/bubbles was examined for each day in relation to the geomagnetic activity of that day, which is taken as the average value of $K_{p}$ recorded during the $6 \mathrm{~h}$ prior to the BSSF onset (e.g. Fejer et al., 1999; Whalen, 2002). Notice that the average $K_{p}$ (hereafter referred simply as $K_{p}$ ) is generally derived from the interval of 18:00-24:00 UT (13:00-19:00 LT). The numbers of BSSF day, digisonde day, bubble day, and ROCSAT- 1 day for each $K_{p}$ in the equinoctial, summer, and winter months are displayed in Tables 2, 3, and 4 , respectively.

The occurrence probabilities of BSSF (black bar) and bubbles (gray bar) observed are categorized by season and plotted versus $K_{p}$ in Fig. 6 . The occurrence probability in each $K_{p}$ for the BSSF (bubble) event is the ratio of BSSF (bubble)
Table 2. Numbers of BSSF day, digisonde day, bubble day, and ROCSAT-1 day for each average $K_{p}$ in the equinoctial months.

\begin{tabular}{lllllllll}
\hline average $K_{p}$ & 0 & 1 & 2 & 3 & 4 & 5 & 6 & 7 \\
\hline BSSF day (days) & 5 & 32 & 16 & 20 & 17 & 2 & 0 & 0 \\
digisonde day (days) & 5 & 33 & 18 & 25 & 22 & 4 & 0 & 1 \\
\hline bubble day (days) & 3 & 9 & 5 & 3 & 2 & 0 & 0 & 0 \\
ROCSAT-1 day (days) & 4 & 15 & 12 & 11 & 14 & 1 & 0 & 0 \\
\hline
\end{tabular}

Table 3. Numbers of BSSF day, digisonde day, bubble day, and ROCSAT-1 day for each average $K_{p}$ in the summer months.

\begin{tabular}{lllllllll}
\hline average $K_{p}$ & 0 & 1 & 2 & 3 & 4 & 5 & 6 & 7 \\
\hline BSSF day (days) & 6 & 18 & 23 & 28 & 14 & 2 & 0 & 0 \\
digisonde day (days) & 6 & 19 & 24 & 28 & 14 & 3 & 1 & 0 \\
\hline bubble day (days) & 1 & 4 & 7 & 6 & 4 & 1 & 0 & 0 \\
ROCSAT-1 day (days) & 5 & 15 & 13 & 23 & 12 & 3 & 1 & 0 \\
\hline
\end{tabular}

Table 4. Numbers of BSSF day, digisonde day, bubble day, and ROCSAT-1 day for each average $K_{p}$ in the winter months.

\begin{tabular}{lllllllll}
\hline average $K_{p}$ & 0 & 1 & 2 & 3 & 4 & 5 & 6 & 7 \\
\hline BSSF day (days) & 9 & 11 & 10 & 8 & 2 & 1 & 0 & 1 \\
digisonde day (days) & 12 & 30 & 26 & 20 & 7 & 2 & 0 & 1 \\
\hline bubble day (days) & 0 & 0 & 0 & 0 & 1 & 0 & 0 & 0 \\
ROCSAT-1 day (days) & 7 & 14 & 12 & 9 & 5 & 2 & 0 & 1 \\
\hline
\end{tabular}

day to digisonde (ROCSAT-1) day. In the equinoctial months (Fig. 6a), BSSF occurs frequently or less frequently when $K_{p}$ is 0 or 5 , respectively. The occurrence probability for a BSSF event tends to decrease with increasing $K_{p}$ in this season. Such a descending trend in occurrence probability for $K_{p}=0$ to 4 also appears for the bubble events. On the other hand, during the summer and winter months, no descending trend from $K_{p}=0$ to 5 is found in the distribution of the BSSF and bubble events (Figs. $6 \mathrm{~b}$ and c). Note that since there was only a single event, the descending trend in the bubble event cannot be identified for the winter months. Based on Fejer et al. (1999) and Whalen (2002), the descending trend in the equinoctial months indicates that increasing the geomagnetic activity would progressively suppress the BSSF and bubble developments in this season. However, these results are different from the report by Whalen (2002) (his Fig. 5), who reported that the descending trends in the total BSSF and macroscopic bubble occurrences are observed 

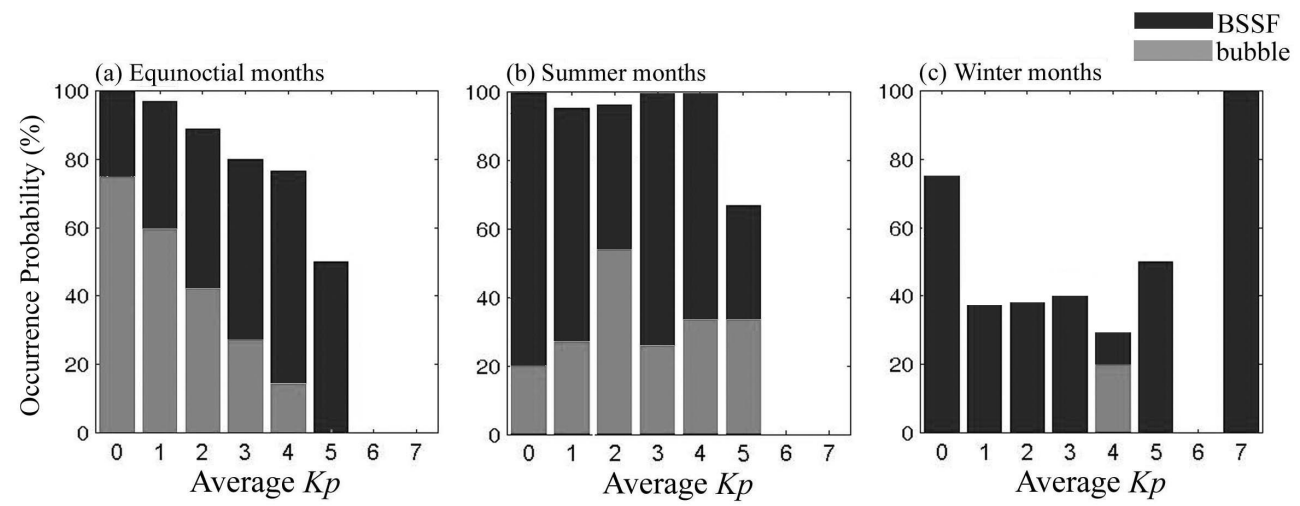

Fig. 6. Occurrence probabilities of BSSF (black bar) and bubble (gray bar) for equinoctial (a), summer (b), and winter (c) months plotted as a function of $K_{p}$ determined as the average during the $6 \mathrm{~h}$ prior to measurement.
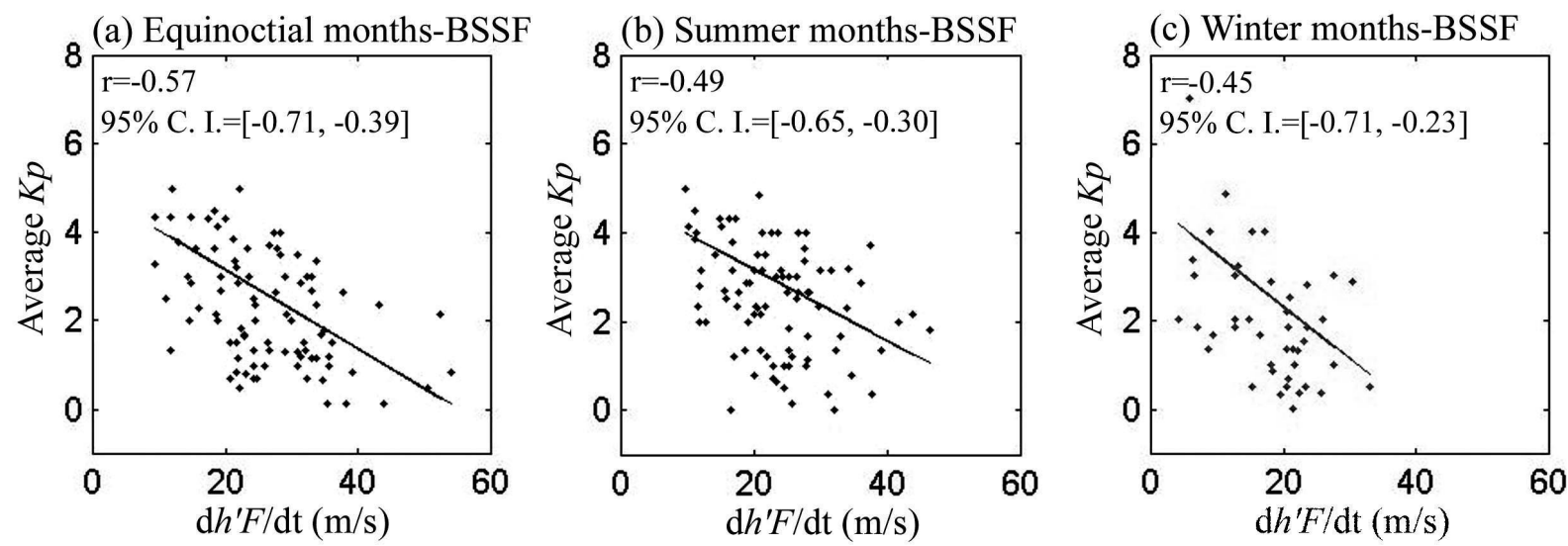

(d) Equinoctial months-bubble
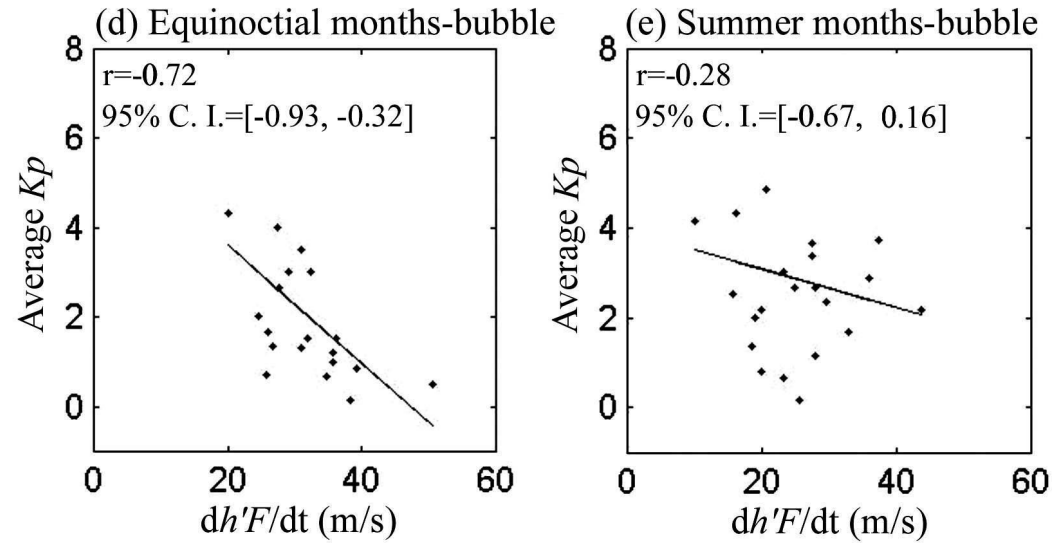

Fig. 7. Scatter plots of $K_{p}$ and $\mathrm{d} h^{\prime} F / \mathrm{dt}$ for the BSSF and bubble events in the equinoctial (a, d), summer (b, e), winter (c) months. The correlation coefficient and $95 \%$ corresponding confidence interval (C. I.) are displayed in the upper-left corner. The solid line is the least-squares straight line fitting to the points.

in the equinoctial and summer months, but not in the winter months. This difference might be related to the geomagnetic effect on the pre-reversal vertical velocity.

Consequently, the scatter plots of $K_{p}$ and $\mathrm{d} h^{\prime} F / \mathrm{dt}$ for the BSSF and bubble events in the equinoctial, summer, and winter months are shown in Fig. 7. In the equinoctial months (Figs. 7a and d), the correlation coefficients
$(-0.57$ and -0.72$)$ and $95 \%$ corresponding confidence intervals $-(-0.71,-0.39)$ and $(-0.93,-0.52)$ mean that $K_{p}$ and $\mathrm{d} h^{\prime} F / \mathrm{dt}$ are moderately and well anti-correlated in the BSSF and bubble events, respectively. Notice that the $95 \%$ corresponding confidence interval is obtained by the Fisher's $z$-transformation with a normal approximation (David, 1938). The least-squares straight lines fitting 
to the points are given by $K_{p}=-0.09 \times \mathrm{d} h^{\prime} F / \mathrm{dt}+4.95$ and $K_{p}=-0.14 \times \mathrm{d} h^{\prime} F / \mathrm{dt}+6.24$ for the BSSF and bubble events, respectively. The moderate and well anti-correlations reveal that the geomagnetic activity in this season would inhibit the maximum pre-reversal velocity, and further suppress the BSSF and bubble generations (Fig. 6a). This suppression effect is due to the disturbance dynamo electric fields which decrease the upward drift velocity near the sunset terminator (Scherliess and Fejer, 1997; Fejer et al., 1999). Moreover, the greater value of the absolute coefficient in Fig. $7 d$ suggests that the suppression effect of geomagnetic activity is more effective for bubble than for BSSF development.

For the summer months, the correlation coefficients $(-0.49$ and -0.28$)$ and the least-squares straight lines $\left(K_{p}=-0.08 \times \mathrm{d} h^{\prime} F / \mathrm{dt}+4.77\right.$ and $\left.K_{p}=-0.04 \times \mathrm{d} h^{\prime} F / \mathrm{dt}+3.93\right)$ of the BSSF and bubble events are displayed in Figs. $7 \mathrm{~b}$ and e. The moderate anti-correlation of -0.49 indicates that the geomagnetic activity could suppress the vertical velocity in the BSSF event. However, the poor anti-correlation $(-0.28)$ indicates that the geomagnetic suppression effect on $\mathrm{d} h^{\prime} F / \mathrm{dt}$ is not evident in the bubble event. Recall that the descending trend is not obvious in Fig. $6 \mathrm{~b}$; the geomagnetic suppression effects on the vertical velocity, and the BSSF and bubble formations are not evident in this season. Similarly, in the winter months (Fig. 7c), the moderate anti-correlation is -0.45 and the least-squares straight line is $K_{p}=-0.12 \times \mathrm{d} h^{\prime} F / \mathrm{dt}+4.66$. Notice that the descending trend in the plot of BSSF days versus $K_{p}$ is not evident in Fig. 6c. Therefore, in this season, the geomagnetic suppression due to the disturbance dynamo electric fields does not affect either the vertical drift velocity or the BSSF generation.

\section{Summary and conclusion}

We have analyzed one year of data of BSSF and bubble observations in the equatorial ionosphere. This analysis is the first attempt to investigate concurrently the equatorial BSSF and bubble occurrences, respectively, obtained by the Jicamarca digisonde and ROCSAT-1 during April 1999-March 2000. The occurrence dependences of BSSF and bubble formation on season, $\mathrm{d} h^{\prime} F / \mathrm{dt}$, and $K_{p}$ were also examined in the work.

For the BSSF events, the higher occurrence probabilities correlate well with a larger mean $\mathrm{d} h^{\prime} F / \mathrm{dt}(>20 \mathrm{~m} / \mathrm{s})$ in the equinoctial and summer months. This result confirms that a larger pre-reversal velocity will lift the F-layer to higher altitudes, and, in turn, help to develop the bottomside F-layer irregularities in these seasons. In contrast, a lower occurrence probability and a smaller vertical velocity in the winter months indicates that the small pre-reversal velocity cannot raise the F-layer high enough to generate irregularities. For the bubble events, the two-peak distribution with maximums in March and October is coincidentally observed in the seasonal variations for both the bubble occurrence and the mean $\mathrm{d} h^{\prime} F / \mathrm{dt}$. This propounds that the bubble generation is associated with the magnitude of the maximum pre-reversal drift velocity.

For the geomagnetic activity, we have demonstrated that the number of days for a BSSF/bubble event decreases with increasing $K_{p}$ in the equinoctial months, but not in the summer and winter months. The fact of this anti-correlation suggests that the geomagnetic activity progressively suppresses the BSSF and bubble developments in that season. Additionally, well to moderate anti-correlations between $K_{p}$ and $\mathrm{d} h^{\prime} F / \mathrm{dt}$ show that the pre-reversal drift velocity could be inhibited by the geomagnetic activity, likely as a result of the disturbance dynamo effects. In contract, the BSSF and bubble generations may not be suppressed by the geomagnetic activity in the summer and winter months.

Acknowledgements. C.-C. Lee was supported by the grant of National Science Council NSC 93-2119-M-231-002 and NSC 942111-M-231-001. S.-Y. Su was supported by the grant of National Science Council NSC 93-2111-M-008-023-AP, and NSC 94NSPO(B)-IPEI-FA07-01. B. W. Reinisch was supported by the grant of AF grant \#F19628-02-C-0092. The authors would like to thank the National Geophysical Data Center (NGDC) (www.ngdc. noaa.gov) for providing data of $K_{p}$, sunspot number, and F10.7 solar flux.

Topical Editor M. Pinnock thanks K. Shiokawa and another referee for their help in evaluating this paper.

\section{References}

Aarons, J.: The longitudinal morphology of equatorial F-layer irregularities relevant to their occurrence, Space Sci, Rev., 63, 209-243, 1993.

Bittencourt, J. A. and Abdu, M. A.: A theoretical comparison between apparent and real verical ionization drift velocities in the equatorial F region, J. Geophys. Res., 86, 2451-2454, 1981.

David, F. N.: Tables of the Correlation Coefficient, in: Tables for Statistics 1976, edited by: Hartley, H. O. and Pearson, E. S., reprinted in Biometrika, vol. I., 3rd edition, Cambridge University Press, Cambridge, 1938.

Fejer, B. G., Scherliess, L., and de Paula, E. R.: Effects of the vertical plasma drift velocity on the generation and evolution of equatorial F, J. Geophys. Res., 104, 19 859-19869, 1999.

Huang, C. Y., Burke, W. J., Machuzak, J. S., Gentile, L. C., and Sultan, P. J.: DMSP observations of equatorial plasma bubbles in the topside ionosphere near solar maximum, J. Geophys. Res., 106, 8131-8142, 2001.

Kelley, M. C.: The Earth's Ionosphere, Int. Geophys. Ser., vol. 43, Academic, San Diego, Calif, 1989.

Lee, C. C., Liu, J. Y., Reinisch, B. W., Chen, W. S., and Chu, F. D.: The effects of the pre-reversal $\boldsymbol{E} \times \boldsymbol{B}$ drift, the EIA asymmetry, and geomagnetic activity on the equatorial spread $\mathrm{F}$ during solar maximum, Ann. Geophys., 23, 745-751, 2005,

SRef-ID: 1432-0576/ag/2005-23-745.

Maruyama, T.: A diagnostic model for equatorial spread F 1, Model description and application to electric field and neutral wind effects, J. Geophys. Res., 93, 14 611-14 622, 1988.

Maruyama, T. and Matuura, N.: Longitudinal variability of annul changes in activity of equatorial spread $\mathrm{F}$ and plasma depletions, J. Geophys. Res., 89, 10 903-10912, 1984. 
Reinisch, B. W.: Modern ionosondes, in: Modern Radio Science, edited by: Kohl, H., Ruester, R., and Schlegel, K., European Geophysical Society, Katlenburg-Lindau, Germany, 440-458, 1996.

Scherliess, L. and Fejer, G. B.: Storm time dependence of equatorial disturbance dynamo zonal electric fields, J. Geophys. Res., 102, 24 037-24 046, 1997.

Su, S. Y., Yeh, H. C., and Heelis, R. A.: ROCSAT-1 IPEI observations of equatorial spread F-some early transitional scale results, J. Geophys. Res., 106, 29 153-29 159, 2001.

Sultan, P. J.: Linear theory and modeling of the Rayleigh-Talor instability leading to the occurrence of equatorial F, J. Geophys. Res., 101, 26 875-26 891, 1996.

Tsunoda, R. T., Livington, R. C., McClure, J. P., and Hanson, W. B.: Equatorial plasma bubbles: Vertical elongated wedges from the bottomside F layer, J. Geophys. Res., 87, 9171-9180, 1982.
Whalen, J. A.: Equatorial bubbles observed at the north and south anomaly crests: Dependence on season, local time and dip latitude, Radio Sci, 32, 1559-1566, 1997.

Whalen, J. A.: Dependence of the equatorial bubbles and bottomside spread $\mathrm{F}$ on season, geomagnetic activity, and $\mathrm{E} \times \mathrm{B}$ drift velocity during solar maximum, J. Geophys. Res., 107, doi:10.1029/2001JA000039, 2002.

Woodman, R. F. and La Hoz, C.: Radar observations of F region equatorial irregularities, J. Geophys. Res., 81, 5447-5466, 1976.

Yeh, H. C., Su, S. Y., Yeh, Y. C., Wu, J. M., Heelis, R. A., and Holt, B. J.: Scientific mission of the IPEI payload onboard ROCSAT-1, Terr. Atmos. Ocean. Sci., suppl., 19-42, 1999.

Zalesack, S. T., Ossalow, S. L., and Chaturvedi, P. K.: Nonlinear equatorial spread F: the effect of neutral winds and background Pederson conductivity, J. Geophys. Res., 87, 151-166, 1982. 